\title{
Bistabilité optique dans un laser à absorbant saturable trimode
}

\author{
S. Djabi, M. Djabi, O. Benkherourou
}

S. Djabi, M. Djabi, O. Benkherourou, "Bistabilité optique dans un laser à absorbant saturable trimode," Proc. SPIE 9664, Ninth International Topical Meeting on Education and Training in Optics and Photonics, 96642S (24 October 2005); doi: 10.1117/12.2207782

SDIE Event: Ninth International Topical Meeting on Education and Training in Optics and Photonics, 2005, Marseille, France 


\title{
Ref ETOP097
}

\section{Bistabilité optique dans un laser à absorbant saturable trimode}

\author{
S.Djabi, M.Djabi, O.Benkherourou
}

Laboratoire des systèmes photoniques et optique non linéaire Département d'optique et de mécanique de précision, Faculté des Sciences de l'ingénieur, Université de Sétif, 19000 Algérie.s_djabi@yahoo.fr

\begin{abstract}
Cette étude est consacrée à l'élaboration d'un modèle mathématique simple pour décrire l'action des lasers à absorbants saturables, l'approche envisagée ici tient compte d'une manière phénoménologique des processus physiques essentiels qui permet de dégager les principaux paramètres physiques dans les LSA et leurs influences sur la bistabilité optique. Nous allons étudier théoriquement la bistabilité optique dans les lasers à absorbants saturables trimodes dans le cas où les deux milieux actif et absorbant subissent un élargissement homogène.

Nous avons établi un programme permettant de déterminer l'effet de la bistabilité optique, de tracer les courbes qui représentent les densités de photons en fonction du pompage du milieu actif et d'analyser la stabilité linéaire des solutions obtenues.
\end{abstract}

\section{Keywords}

bistabilité optique, laser à absorbant saturable, élargissement homogène, cavité Fabry-Pérot

\section{1- Introduction}

\section{Summary}

La bistabilité optique a connu un grand développement ces dernières années. La plupart des bistables optiques développés jusqu'à présent font intervenir deux états de transmission qui se traduisent par des niveaux "haut" et" bas" de l'énergie transmise par le dispositif.

Les systèmes optiques bistables ont des applications importantes dans les ordinateurs et les télécommunications optiques parce qu'ils permettent de réaliser des portes logiques, des commutateurs, des amplificateurs et d'autres éléments dont l'action est contrôlable et très rapide. La possibilité de développer des transistors optiques et d'une façon générale des composants optiques quelconques permet d'envisager des ensembles de traitement optiques.

Pour prévoir le phénomène de la bistabilité optique, il est nécessaire d'introduire des équations non linéaires pour décrire ce phénomène d'hystérésis.

La structure d'un laser à absorbant saturable est donnée par la Fig1 :

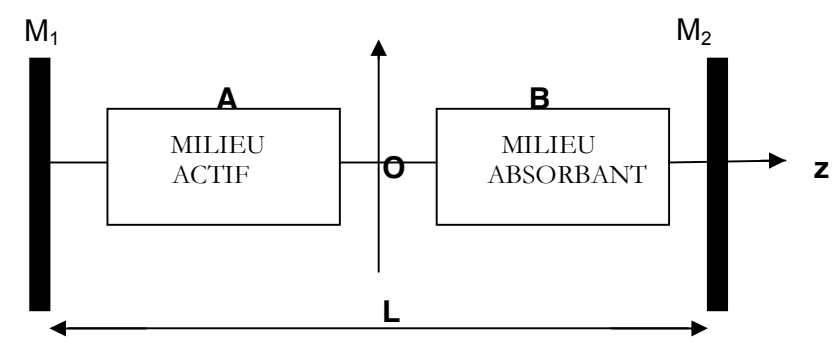

$\mathrm{M}_{1}, \mathrm{M}_{2}$ : Miroirs plans 


\section{$\mathrm{L}$ :longueur de la cavité}

Fig.1: Structure d'un laser à absorbant saturable

\section{Modèle mathématique}

Le système d'équations d'un laser à absorbant saturable trimode comprend quatre équations : une équation donnant la densité de photons dans la cavité résonnante du mode central,une équation donnant la densité de photons dans la cavité résonnante du mode d'ordre \pm 1 , une équation de bilan de la variation de la différence de population du milieu actif et une équation de bilan de la variation de la différence de population du milieu absorbant. II s'écrit de la façon suivante [1,2] :

$$
\left.\begin{array}{l}
\frac{d n_{0}}{d t}=-x_{0} n_{0}+B L g_{0}\left(n_{0}+1\right)\left[\int_{z 2-\frac{l}{2}}^{z 1+\frac{l}{2}} N_{a} \sin 2\left(\frac{\pi}{L} q z\right) d z-\int_{z 2-\frac{l}{2}}^{z 1+\frac{l}{2}} N_{b} \sin 2\left(\frac{\pi}{L} q z\right) d z\right] \\
: \frac{d n_{ \pm 1}}{d t}=-x_{ \pm 1} n_{ \pm 1}+B L g_{ \pm 1}\left(n_{ \pm 1}+1\right)\left[\int_{z 2-\frac{l}{2}}^{z 1+\frac{l}{2}} N_{a} \sin 2\left(\frac{\pi}{L} q z\right) d z-\int_{z 2-\frac{l}{2}}^{z 1+\frac{l}{2}} N_{b} \sin 2\left(\frac{\pi}{L} q z\right) d z\right] \\
\frac{d N_{a}}{d t}=\frac{R_{a}}{L}-N_{a}\left(B g_{0} n_{0}+2 B g_{ \pm 1} n_{ \pm 1}+\gamma_{a}\right) \\
\frac{d N_{b}}{d t}=\frac{R_{b}}{L}-N_{b}\left(B g_{0} n_{0+} 2 B g_{ \pm 1} n_{ \pm 1}+\gamma_{b}\right)
\end{array}\right\}
$$

Où :

$n_{ \pm 1}, n_{0}$ sont respectivement les densités de photons dans la cavité pour le mode $j=0$ et $j= \pm 1$.

$N_{a}, N_{b}$ sont respectivement les densités d'inversion de population du milieu actif (A) et du milieu absorbant (B).

$x_{ \pm 1}, x_{0}$ sont respectivement les coefficients des pertes du résonateur pour le mode $j=0$ et $j= \pm 1$

$B$ est le coefficient d'Einstein ; $L$ est longueur de la cavité ; $q$ est le nombre de mode.

$\gamma_{a}, \gamma_{b}$ sont respectivement les coefficients du relaxation du milieu actif et absorbant.

Où : $\quad \gamma_{b}=\xi \gamma_{a}=\xi \gamma$ avec $\xi$ qui est coefficient de saturation $(0 \leq \xi \leq 1)$

Le profil de Lorentz g est défini par : $\quad g=\frac{\Gamma^{2}}{\Gamma^{2}+\Delta^{2}{ }_{j}}$ 
Où : $\Gamma$ est largeur de raie homogène. et $\quad \Delta_{\mathrm{j}}=\left|\omega_{j}-\omega_{0}\right|$

avec : $\omega_{j}$ est la fréquence d'ordre j et $\omega_{0}$ est la fréquence centrale.

$R_{a}, R_{b}$ sont respectivement le pompage du milieu actif et du milieu absorbant,

$\left[\int_{z_{2}-\frac{l}{2}}^{z_{1}+\frac{l}{2}} N_{a} \sin ^{2}\left(\frac{\pi}{L} q z\right) d z-\int_{z_{2}-\frac{l}{2}}^{z_{1}+\frac{l}{2}} N_{b} \sin ^{2}\left(\frac{\pi}{L} q z\right) d z\right]:$ ce terme entre crochets désigne l'effet

d'interférence produit dans le résonateur Fabry-Pérot. II peut prendre différentes valeurs qui dépendent de la position des deux milieux actif et absorbant dans le résonateur optique.

Dans le cas général, les populations $\mathrm{N}_{\mathrm{a}}$ et $\mathrm{N}_{\mathrm{b}}$ sont indépendantes de la position $z$ et par conséquent ce terme entre crochets peut s'écrire de la façon suivante:

$\left(N_{a}-N_{b}\right)\left[\int_{z_{2}-\frac{l}{2}}^{z_{1}+\frac{l}{2}} \sin ^{2}\left(\frac{\pi}{L} q z\right) d z\right]=\left(N_{a}-N_{b}\right) \frac{A_{0}}{2}$

Pour le cas où les milieux actif et absorbant sont placés au milieu de la cavité, $A_{0}$ prend la valeur $\left[1-\frac{L}{\pi q} \quad \sin \frac{\pi q l}{L}\right]$ où $L$ est la longueur de la cavité , I est la longueur du milieu actif ou absorbant

\section{Effet de la bistabilité optique}

Dans notre cas, nous étudions le phénoméne de la bistabilité optique et de ce fait, nous nous intéressons seulement au cas stationnaire laser c'est-à-dire les dérivées des variables par rapport aux temps sont nulles et par conséquent, nous posons à cet effet :

$\frac{d n_{ \pm 1}}{d t}=\frac{d n_{0}}{d t}=\frac{d N a}{d t}=\frac{d N b}{d t}=0$

A partir des deux premières équations du systéme (1), nous obtenons la relation qui est donnée par :

$$
\frac{x_{0} n_{0}}{x_{ \pm 1} n_{ \pm 1}}=\frac{g_{0}\left(n_{0}+1\right)}{g_{ \pm 1}\left(n_{ \pm 1}+1\right)}
$$

et pour faciliter les calculs, plusieurs changements de variables ont été utilisés:

$$
Q_{j}=\frac{B}{\gamma} n_{j}, \quad \sigma_{a j}=\frac{B}{\gamma} \frac{R a}{x_{j}}, \quad \text { Avec }: j=0, \pm 1
$$

Selon l'équation (2), on distingue deux cas : 
3.1 Cas où : $x_{0} g_{ \pm 1}-x_{ \pm 1} g_{0}=0$

Si ce terme est nul cela revient à négliger le terme 1 du système d'équations (1) c'est-àdire nous ne tinons pas compte de l'émission spontanée.

Nous étudions les cas où le coefficient de saturation est égal à l'unité $(\xi=1)$ et $\xi$ est différent de l'unité $(\xi \neq 1)$.

a) Cas où le coefficient de saturation égal à l'unité $(\xi=1)$

Nous injectons cette relation dans l'équation (1) et après plusieurs transformations, nous obtenons une équation algébrique du deuxième degré de la forme:

$$
Q_{0}^{2}+Q_{0} A_{1}+B_{2}=0
$$

Où : $A_{2}=\frac{1}{g_{0}+2 g_{ \pm 1}}-\frac{A_{0} g_{0}\left(\sigma_{a 0}-\sigma_{b 0}\right)}{2\left(g_{0}+2 g_{ \pm 1}\right)} \quad$ et $\quad B_{2}=-\frac{A_{0} g_{0}\left(\sigma_{a 0}-\sigma_{b 0}\right)\left(\frac{B}{\gamma}\right)}{2\left(g_{0}+2 g_{ \pm 1}\right)}$.

Les conditions d'apparition de la BO ne sont pas vérifiées et par suite l'effet de la BO n'existe pas.

b) Cas où le coefficient de saturation est différent de l'unité $(\xi \neq 1)$

En procédant de la même manière que précédemment, nous trouvons une équation du troixième degré de la forme :

$Q_{0}^{3}+A_{3} Q_{0}^{2}+B_{3} Q_{0}+C_{3}=0$.

Où :

$$
\begin{aligned}
& A_{3}=\frac{(1+\xi) x_{ \pm 1}}{g_{0} x_{ \pm 1}+4 x_{ \pm 1} g_{ \pm 1}+4 \frac{g_{ \pm 1}^{3}}{g_{0}^{2}} x_{0}}-\frac{A_{0}\left(\sigma_{a 0}-\sigma_{b 0}\right)}{1+4 \frac{x_{ \pm 1}}{x_{0}}+4 \frac{g_{ \pm 1}^{2}}{g_{0}^{2}}}+\frac{2(1+\xi) x_{ \pm 1} \frac{g_{ \pm 1}}{g_{0}}}{g_{0} x_{ \pm 1}+4 x_{ \pm 1} g_{ \pm 1}+4 \frac{g_{ \pm 1}^{3}}{g_{0}^{2}} x_{0}}-\frac{A_{0}\left(\sigma_{a 0}-\sigma_{b 0}\right) x_{ \pm 1} g_{ \pm 1}}{g_{0} x_{ \pm 1}+4 x_{ \pm 1} g_{ \pm 1}+4 \frac{g_{ \pm 1}^{3}}{g_{0}^{2}} x_{0}} . \\
& B_{3}=\frac{\frac{\xi}{g_{0}^{2}}}{1+4 \frac{x_{ \pm 1}}{x_{0}}+4 \frac{A_{ \pm 1}^{2}}{g_{0}^{2}}\left(\sigma_{a_{0}}-\sigma_{b_{0}}\right)\left(\frac{B}{\gamma}\right)}-\frac{\frac{A_{0}}{2}\left(\sigma_{a_{0}} \xi-\sigma_{b_{0}}\right) x_{ \pm 1}}{1+4 \frac{x_{ \pm 1}}{x_{0}}+4 \frac{g_{ \pm 1}^{2}}{g_{0}^{2}}}-\frac{A_{0}\left(\sigma_{a_{0}}-\sigma_{b_{0}}\right)\left(\frac{B}{\gamma}\right) x_{ \pm 1} g_{ \pm 1}}{g_{0} x_{ \pm 1}+4 x_{ \pm 1} g_{ \pm 1}+4 \frac{g_{ \pm 1}^{2}}{g_{0}^{2}} x_{0}} g_{0} x_{ \pm 1}+4 x_{ \pm 1} g_{ \pm 1}+4 \frac{g_{ \pm 1}^{3}}{g_{0}^{2}} x_{0}
\end{aligned}
$$

$$
C_{3}=-\frac{\frac{A_{0}}{2}\left(\sigma_{a 0} \xi-\sigma_{b 0}\right) x_{ \pm 1}\left(\frac{B}{\gamma}\right)}{g_{0} x_{ \pm 1}+4 x_{ \pm 1} g_{ \pm 1}+4 \frac{g_{ \pm 1}^{3}}{g_{0}^{2}} x_{0}} .
$$

Puisque le dernier terme de l'équation (5) est très petit devant l'unité $\left(B / \gamma=10^{-11}\right)$ donc la méthode de Greaff- Lobatchevski peut être appliquée ; l'équation ( 5) prend alors la forme suivante : 


$$
\left(Q_{0}+\frac{c_{3}}{B_{3}}\right)\left(Q_{0}^{2}+A_{3} Q_{0}+B_{3}\right)=0
$$

Les solutions de cette équation sont facilement déterminées

3.2. Cas où : $x_{0} g_{ \pm 1}-x_{ \pm 1} g_{0} \neq 0$

Dans ce cas, nous tenons compte de l'émission spontanée. De la même façon, nous prenons les cas où le coefficient de saturation est égal à l'unité et différent de l'unité

\subsubsection{Cas où le coefficient de saturation est égal à l'unité $(\xi=1)$}

Nous obtenons après de simples transformations, une équation du troisième degré en $Q_{0}$ de la forme suivante :

$$
Q_{0}^{3}+A Q_{0}^{2}+B Q_{0}+C=0
$$

Où:

$$
\begin{aligned}
& A=\frac{\left(\frac{B}{\gamma}\right) g_{0} x_{ \pm 1}}{\left(x_{ \pm 1} g_{0}-x_{0} g_{ \pm 1}\right)}-\frac{2\left(\frac{B}{\gamma}\right) x_{0} g_{ \pm 1}^{2}}{g_{0}\left(x_{ \pm 1} g_{0}-x_{0} g_{ \pm 1}\right)}+\frac{1}{g_{0}}-\frac{A_{0}}{2}\left(\sigma_{a_{0}}-\sigma_{b_{0}}\right) . \\
& B=\frac{\left(\frac{B}{\gamma}\right) x_{ \pm 1} \quad}{\left(x_{ \pm 1} g_{0}-x_{0} g_{ \pm 1}\right)}-\frac{A_{0} g_{0} x_{ \pm 1}\left(\sigma_{a_{0}}-\sigma_{b 0}\right)\left(\frac{B}{\gamma}\right)}{2\left(x_{ \pm 1} g_{0}-x_{0} g_{ \pm 1}\right)}-\left(\frac{A_{0}}{2}\right)\left(\frac{B}{\gamma}\right)\left(\sigma_{a_{0}}-\sigma_{b_{0}}\right) . \\
& C=-\left(\frac{A_{0}}{2}\right) \quad\left(\frac{B}{\gamma}\right)^{2} g_{0} \frac{\left(\sigma_{a_{0}}-\sigma_{b_{0}}\right)}{\left(x_{ \pm 1} g_{0}-x_{ \pm 1} g\right)} .
\end{aligned}
$$

En résumé, pour trouver au moins deux solutions positives c'est à dire l'effet de la bistabilité optique, il faut que le pompage du milieu actif $\left(\sigma_{a_{0}}\right)$ doit satisfaire aux conditions suivantes :

$$
\left\{\begin{array}{l}
\sigma_{a_{0}} \prec \sigma_{a_{01}}=\frac{2 x_{ \pm 1}}{A_{0}\left(2 x_{ \pm 1} g_{0}-x_{0} g_{ \pm 1}\right)}+\sigma_{b_{0}} \\
\sigma_{a_{0}} \succ \sigma_{a_{02}}=\frac{2 x_{ \pm 1}\left(g_{0}\left(\frac{B}{\gamma}\right)+1\right)+2 x_{0}\left(2\left(\frac{B}{\gamma}\right) \frac{g_{ \pm 1}^{2}}{g_{0}}-\frac{g_{ \pm 1}}{g_{0}}\right)}{A_{0}\left(x_{ \pm 1} g_{0}-x_{0} g_{ \pm 1}\right)}+\sigma_{b_{0}} \\
\sigma_{a_{0}} \prec \sigma_{a_{03}}=\frac{2 x_{ \pm 1}}{A_{0}\left(2 x_{ \pm 1} g_{0}-x_{0} g_{ \pm 1}\right)}+\sigma_{b_{0}}
\end{array}\right.
$$

Nous constatons que les conditions ne sont pas vérifiées à la fois et par conséquent il n'y a pas de bistabilité optique dans le cas où le coefficient de saturation $\xi=1$.

3.2.2. Cas où le coefficient de saturation est différent de l'unité $(\xi \neq 1)$ 
Avec les mêmes étapes de calcul et utilisant les expressions de $Q_{0}, Q_{ \pm 1}, \sigma_{a 0}$ et $\sigma_{b_{0}}$, nous obtenons une équation algébrique de cinquiéme degré de la forme suivante :

$$
Q_{0}^{5}+A_{1} Q_{0}^{4}+B_{1} Q_{0}^{3}+C_{1} Q_{0}^{2}+D_{1} Q_{0}+E_{1}=0
$$

Où :

$$
\begin{aligned}
& A_{1}=\frac{2 g_{ \pm 1}\left(\frac{B}{\gamma}\right) x_{ \pm 1}}{\left(x_{ \pm 1} g_{0}-x_{0} g_{ \pm 1}\right)}+\frac{4 g_{ \pm 1}^{2} x_{0}\left(\frac{B}{\gamma}\right)}{g_{0}\left(x_{ \pm 1} g_{0}-x_{0} g_{ \pm 1}\right)}+\frac{(1+\xi)}{g_{0}}-\frac{A_{0}}{2}\left(\sigma_{a 0}-\sigma_{b 0}\right) \\
& B_{1}=\frac{g_{0}^{2} x_{ \pm 1}^{2}\left(\frac{B}{\gamma}\right)^{2}}{\left(x_{ \pm 1} g_{0}-x_{0} g_{ \pm 1}\right)^{2}}+\frac{4 g_{ \pm 1}^{2} x_{ \pm 1} x_{0}\left(\frac{B}{\gamma}\right)^{2}}{\left(x_{ \pm 1} g_{0}-x_{0} g_{ \pm 1}\right)^{2}}+\frac{2 x_{ \pm 1}\left(\frac{B}{\gamma}\right)(1+\xi)}{\left(x_{ \pm 1} g_{0}-x_{0} g_{ \pm 1}\right)}-\frac{A_{0} g_{0} x_{ \pm 1}^{2}\left(\frac{B}{\gamma}\right)\left(\sigma_{a 0}-\sigma_{b 0}\right)}{\left(x_{ \pm 1} g_{0}-x_{0} g_{ \pm 1}\right)}+\frac{4 g_{ \pm 1}^{4} x_{0}^{2}\left(\frac{B}{\gamma}\right)^{2}}{\left(x_{ \pm 1} g_{0}-x_{0} g_{ \pm 1}\right)^{2}}+\frac{\xi}{g_{0}^{2}} \\
& +\frac{2 g_{ \pm 1}^{2}(1+\xi) x_{0}\left(\frac{B}{\gamma}\right)}{\left(x_{ \pm 1} g_{0}-x_{0} g_{ \pm 1}\right) g_{0}^{2}}-\frac{A_{0} g_{ \pm 1}^{2} x_{0}\left(\frac{B}{\gamma}\right)\left(\sigma_{a 0}-\sigma_{b 0}\right)}{g_{0}\left(x_{ \pm 1} g_{0}-x_{0} g_{ \pm 1}\right)}-\frac{A_{0}\left(\sigma_{a 0} \xi-\sigma_{b 0}\right)}{2 g_{0}}-\frac{A_{0}}{2}\left(\frac{B}{\gamma}\right)\left(\sigma_{a 0}-\sigma_{b 0}\right) \\
& C_{1}=\frac{(1+\xi) g_{0} x_{ \pm 1}^{2}\left(\frac{B}{\gamma}\right)^{2}}{\left(x_{ \pm 1} g_{0}-x_{0} g_{ \pm 1}\right)^{2}}-\frac{A_{0} g_{0}^{2} x_{ \pm 1}^{2}\left(\frac{B}{\gamma}\right)^{2}\left(\sigma_{a 0}-\sigma_{b 0}\right)}{2\left(x_{ \pm 1} g_{0}-x_{0} g_{ \pm 1}\right)^{2}}+\frac{2(1+\xi) g_{ \pm 1}^{2} x_{0} x_{ \pm 1}\left(\frac{B}{\gamma}\right)^{2}}{\left(x_{ \pm 1} g_{0}-x_{0} g_{ \pm 1}\right) g_{0}}+\frac{2 \xi x_{ \pm 1}\left(\frac{B}{\gamma}\right)}{g_{0}\left(x_{ \pm 1} g_{0}-x_{0} g_{ \pm 1}\right)} \\
& -\frac{A_{0} g_{ \pm 1}^{2} x_{0} x_{ \pm 1}\left(\frac{B}{\gamma}\right)^{2}\left(\sigma_{a 0}-\sigma_{b 0}\right)}{\left(x_{ \pm 1} g_{0}-x_{0} g_{ \pm 1}\right)^{2}}-\frac{A_{0} x_{ \pm 1}\left(\frac{B}{\gamma}\right)\left(\sigma_{a 0} \xi-\sigma_{b 0}\right)}{\left(x_{ \pm 1} g_{0}-x_{0} g_{ \pm 1}\right)}-\frac{A_{0} g_{0} x_{ \pm 1}\left(\frac{B}{\gamma}\right)^{2}\left(\sigma_{a 0}-\sigma_{b 0}\right)}{2\left(x_{ \pm 1} g_{0}-x_{0} g_{ \pm 1}\right)^{2}}-\frac{A_{0} g_{ \pm 1}^{2} x_{0}\left(\frac{B}{\gamma}\right)^{2}\left(\sigma_{a 0}-\sigma_{b 0}\right)}{g_{0}\left(x_{ \pm 1} g_{0}-x_{0} g_{ \pm 1}\right)^{2}}
\end{aligned}
$$

$$
\frac{A_{0}\left(\frac{B}{\gamma}\right)\left(\sigma_{a 0} \xi-\sigma_{b 0}\right)}{2 g_{0}\left(x_{ \pm 1} g_{0}-x_{0} g_{ \pm 1}\right)^{2}} \text {. }
$$$$
D_{1}=\frac{\xi\left(\frac{B}{\gamma}\right)^{2} x_{ \pm 1}^{2}}{\left(x_{ \pm 1} g_{0}-x_{0} g_{ \pm 1}\right)^{2}}-\frac{g_{0} A_{0}\left(\sigma_{a 0} \xi-\sigma_{b 0}\right)\left(\frac{B}{\gamma}\right)^{2} x_{ \pm 1}^{2}}{2\left(x_{ \pm 1} g_{0}-x_{0} g_{ \pm 1}\right)^{2}}-\frac{g_{0}^{2} A_{0}\left(\sigma_{a 0}-\sigma_{b 0}\right)\left(\frac{B}{\gamma}\right)^{3} x_{ \pm 1}^{2}}{2\left(x_{ \pm 1} g_{0}-x_{0} g_{ \pm 1}\right)^{2}}-\frac{g_{ \pm 1}^{2} A_{0}\left(\sigma_{a 0}-\sigma_{b 0}\right)\left(\frac{B}{\gamma}\right)^{3} x_{ \pm 1} x_{0}}{\left(x_{ \pm 1} g_{0}-x_{0} g_{ \pm 1}\right)^{2}}
$$$$
-\frac{A_{0}\left(\sigma_{a 0} \xi-\sigma_{b 0}\right)\left(\frac{B}{\gamma}\right)^{2} x_{ \pm 1}}{\left(x_{ \pm 1} g_{0}-x_{0} g_{ \pm 1}\right)}
$$$$
E_{1}=\frac{-A_{0}\left(\frac{B}{\gamma}\right)^{3} g_{0} x_{ \pm 1}^{2}\left(\sigma_{a 0} \xi-\sigma_{b 0}\right)}{2\left(x_{ \pm 1} g_{0}-x_{0} g_{ \pm 1}\right)^{2}}:
$$ 
3.3. Courbes d'évolution de la densité de photons $Q_{0}$ en fonction du pompage du milieu

actif

Pour préciser les conditions d'apparition de la bistabilité optique, il est commode d'établir un programme qui permet de déterminer les intervalles de la bistabilité optique en se basant sur les conditions de l'existence d'au moins deux solutions positives et de calculer les valeurs de $\left(Q_{01} Q_{02} Q_{03}\right)$ pour différentes valeurs des paramétres $\sigma_{b 0} A_{0}, g_{ \pm 1}, g_{0}, \xi$, en prenant comme variable $\left(\sigma_{a 0}\right)$. Puis, nous avons tracé les courbes d'évolution représentant les densités de photons en fonction du pompage du milieu $\operatorname{actif}\left(\sigma_{a 0}\right)$ pour les paramètres $\sigma_{b 0}, A_{0}, g_{ \pm 1}, g_{0}$ et $\xi$ (Fig.2).et (Fig3)

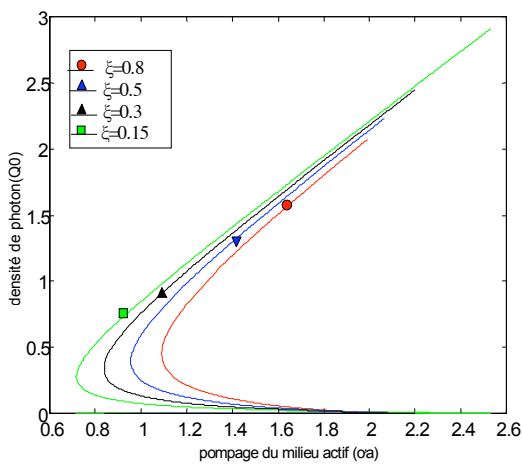

(a) dans le cas où $\xi$ varie

varie.

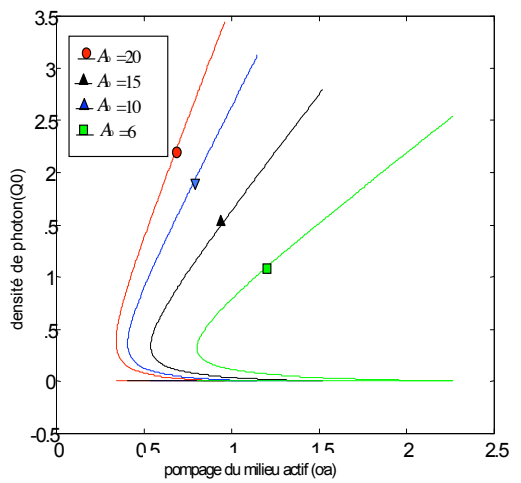

(c) dans le cas où $A_{0}$ varie

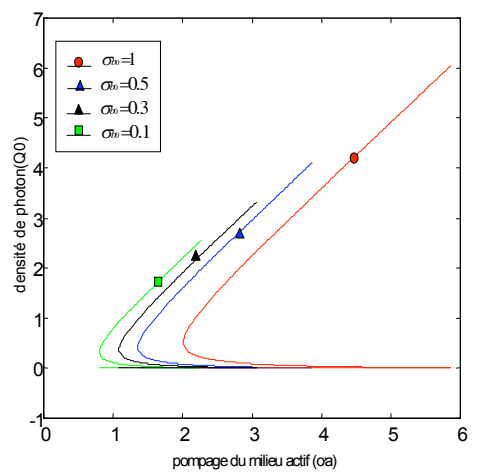

(b) dans le cas où $\sigma_{b o}$

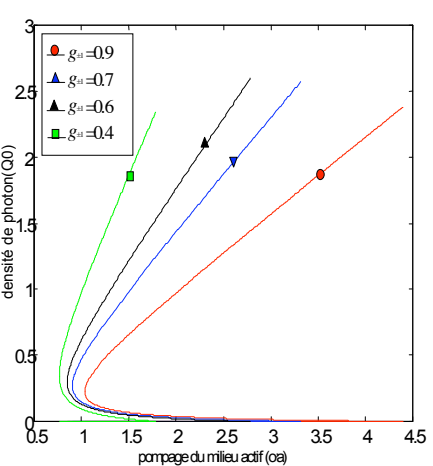

(d) dans le cas où go varie.

Fig. 2. Courbes d'évolution représentant la densité de photons $Q_{0}$ en fonction du pompage du milieu actif $\left(\sigma_{a 0}\right)$ ( cas où l'on ne tient pas compte de l'émission spontanée) 


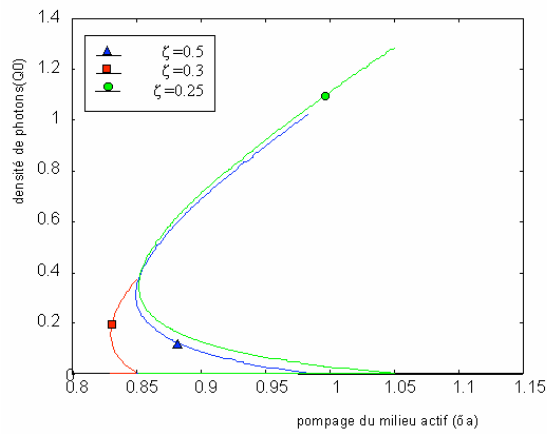

(a)

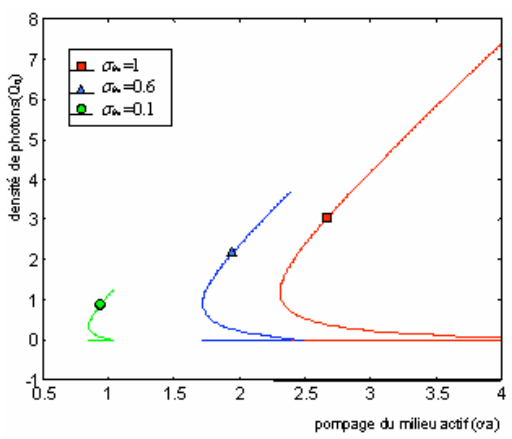

(c)

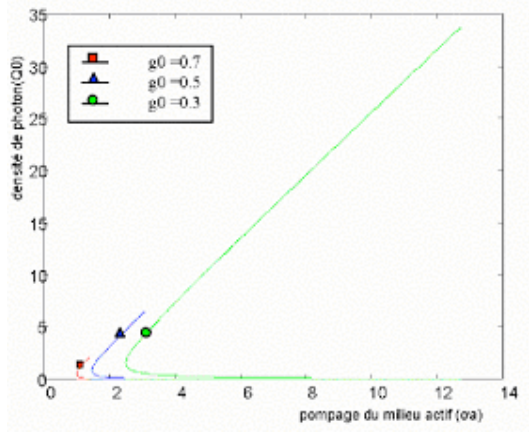

(b)

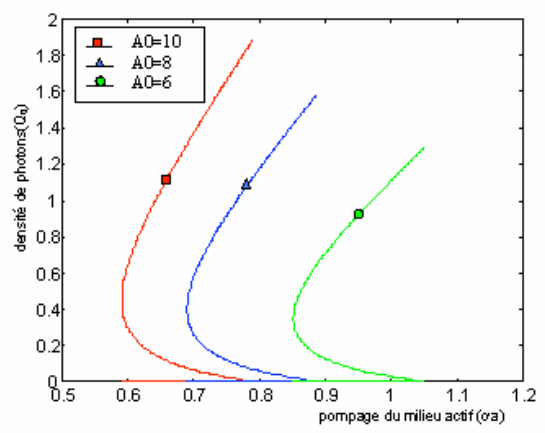

(d)

Fig. 3. Courbes d'évolution de la densité de photons pour différents paramètres. (Cas où l'on tient compte de l'émission spontanée)

3.4. Discussion

Proc. of SPIE Vol. $966496642 S-8$ 
En examinant l'influence des paramètres physique du L.S.A sur l'effet de la bistabilité optique, nous remarquons qu'une diminution du coefficient de saturation, entraîne une augmentation de l'intervalle de l'effet de la bistabitité optique et l'étendue de la densité de photons. Ceci peut être expliqué de la façon suivante : la différence d'énergie du milieu absorbant doit correspondre à la fréquence de transition du laser dans le milieu actif. A l'état initial, l'absorbant saturable est à son opacité maximale c'est -à -dire l'état est non saturé.

L'irradiation lumineuse du filtre à la fréquence d'oscillation donne lieu à des processus d'absorption par résonance et l'émission spontanée c'est-à-dire lorsque le pompage du milieu absorbant $\left(\sigma_{b 0}\right)$ augmente, la saturation est maximale. Après la fin de l'irradiation, les processus de relaxation assurent le retour à l'état fondamental ; l'absorbant reprend son état initial (non saturé). Par contre, si le pompage du milieu absorbant augmente, il provoque ainsi la saturation du milieu et engendre un accroissement de l'intervalle de l'effet de la bistabilité optique et par conséquent l'accroissement de l'étendue de la densité de photons. Pour le paramètre $\left(A_{0}\right)$ : Si $\left(A_{0}\right)\left(A_{0} \approx l\right)$ augmente, nous remarquons une augmentation de l'intervalle de l'effet de la bistabilité optique ainsi que l'étendue de la densité de photons. Cela est dû probablement à la croissance des ondes interférées qui produit une augmentation de l'intensité dans la cavité. Pour les profils de raies $\left(g_{0}, g_{ \pm 1}\right)$ : l'accroissement

de $\left(g_{0}, g_{ \pm 1}\right)$ (soit par l'augmentation de $\Gamma$ ou par la diminution de $\Delta_{j}=\omega_{j}-\omega_{0} \quad$ ) entraîne des augmentations de l'intervalle de l'effet de la bistabilité optique et de l'étendue de la densité de photons. Ceci peut être expliqué par : dans le cas où $\Gamma$ augmente c'est- à -dire il y a beaucoup de modes qui peuvent osciller. Cependant dans le cas où $\Delta_{j}$ diminue, le laser tend à devenir monomode c'est -à -dire $\omega_{j}$ se rapproche de $\omega_{0}$.

\section{Linéarisation du système d'équations non linéaire au voisinage de la solution stationnaire}

L'analyse de stabilité linéaire consiste à déterminer l'évolution de petites variations des équilibres. Si l'équilibre est stable, ces variations s'amortissent dans le temps, elles divergent dans le cas d'un équilibre instable.

On linéarise ce système d'équation différentiel au voisinage des solutions stationnaires, c'est-à-dire que l'on pose :

$$
\begin{gathered}
n_{0}=n_{0 s}+\Delta n_{0}(t) \\
n_{ \pm 1}=n_{ \pm 1 s}+\Delta n_{ \pm 1}(t) \\
N_{a}=N_{a s}+\Delta N_{a}(t) \\
N_{b}=N_{b s}+\Delta N_{b}(t)
\end{gathered}
$$

Où :

$n_{0 s}, n_{ \pm 1 s}, N_{a s}, N_{b s}:$ sont des valeurs stationnaires. 
$\Delta n_{0}, \Delta n_{ \pm 1}, \Delta N_{a}, \Delta N_{b} \quad$ : sont des petits écarts de quantité correspondantes vis à vis de leurs valeurs stationnaires.

En remplaçant $n_{0}, n_{ \pm 1}, N_{a}$ et $N_{b}$ dans les équations du système (1) et en ne gardant dans les équations que les termes en $n_{0 s}, n_{ \pm 1 s}, N_{a s}, N_{b s}$ satisfont au système d'équations (1) et en négligeant les produits de type $\left(\Delta n_{0} \Delta N_{a}\right)$ et $\left(\Delta n_{ \pm 1} \Delta N_{a}\right),\left(\Delta n_{0} \Delta N_{b}\right)$ et $\left(\Delta n_{ \pm 1} . \Delta N_{b}\right)$ qui sont des quantités du second ordre de petitesse, on obtient finalement un système d'équations différentielles linéaires par rapport aux fonctions $\Delta n_{0}(t), \Delta n_{ \pm 1}(t), \Delta N_{a}(t), \Delta N_{b}(t)$ qui est donné par :

$\left[\begin{array}{l}\frac{d \Delta n_{0}}{d t} \\ \frac{d \Delta n_{ \pm 1}}{d t} \\ \frac{d \Delta N_{a}}{d t} \\ \frac{d \Delta N_{b}}{d t}\end{array}\right]=\mathrm{A}\left[\begin{array}{l}n_{0} \\ n_{ \pm 1} \\ N_{a} \\ N_{b}\end{array}\right] \quad$ où $\quad \mathrm{A}==\left[\begin{array}{l}a_{11} a_{12} a_{13} a_{14} \\ a_{21} a_{22} a_{23} a_{24} \\ a_{31} a_{32} a_{33} a_{34} \\ a_{41} a_{42} a_{43} a_{44}\end{array}\right]$

A : La matrice représentant l'équation caractéristique du système d'équations différentielles linéaires

Avec:

$$
\begin{aligned}
& a_{11}=-x_{0}+\frac{B 1 g_{0}}{2} A_{0}\left(N_{a s}-N_{b s}\right), a_{13}=\left(n_{0 s}+1\right) \frac{B l}{2} g_{0} A_{0}=-a_{14}, a_{22}=-x_{ \pm 1}+\frac{B l g_{ \pm 1}}{2} A_{0}\left(N_{a s}-N_{b s}\right) . \\
& a_{12}=a_{21}=a_{34}=a_{43}=0 . \\
& a_{23}=\left(n_{ \pm 1 s}+1\right) \frac{B l}{2} g_{ \pm 1} A_{0}=-a_{24}, a_{23}=\left(n_{ \pm 1 s}+1\right) \frac{B l}{2} g_{ \pm 1} A_{0}=-a_{24}, a_{23}=\left(n_{ \pm 1 s}+1\right) \frac{B l}{2} g_{ \pm 1} A_{0}=-a_{24} . \\
& a_{31}=-N_{a s} B g_{0}, a_{32}=-2 B g_{ \pm 1} N_{a s}, a_{33}=-\left(B g_{0} n_{0 s}+2 B g_{0} n_{ \pm 1 s}+\gamma_{a}\right) . \\
& a_{41}=-N_{b s} B g_{0}, a_{42}=-2 B g_{ \pm 1} N_{b s}, a_{44}=\left(B g_{0} n_{0 s}+2 B g_{0} n_{ \pm 1 s}+\gamma_{b}\right),
\end{aligned}
$$

Calculons le déterminant de $(A+I \lambda)$

Où : $\lambda$ est la valeur propre de la matrice $A$ et I est la matrice identité d'ordre quatre.

On obtient une équation caractéristique du quatrième degré en $\lambda$ de la forme suivante : 


$$
\lambda^{4}+k_{1} \lambda^{3}+k_{2} \lambda^{2}+k_{3} \lambda+k_{4}=0 .
$$

Avec :

$$
\begin{aligned}
& k_{1}=a_{11}+a_{22}+a_{33}+a_{44} . \\
& k_{2}=a_{11}\left(a_{33}+a_{22}+a_{44}\right)+a_{22} a_{44}+a_{33} a_{44}+a_{22} a_{33}-a_{23} a_{32}-a_{24} a_{42}-a_{13} a_{31}-a_{14} a_{41} . \\
& k_{3}=a_{11} a_{22} a_{44}+a_{11} a_{33} a_{44}+a_{22} a_{33} a_{11}-a_{23} a_{32} a_{11}-a_{24} a_{42} a_{11}+a_{22} a_{33} a_{44}-a_{23} a_{32} a_{44} \\
& -a_{24} a_{42} a_{33}-a_{31}\left(a_{22}+a_{44}\right) a_{13}-a_{41} a_{41}\left(a_{22}+a_{33}\right) \\
& k_{4}=a_{22} a_{33} a_{44} a_{11}-a_{23} a_{32} a_{44} a_{11}-a_{24} a_{42} a_{33} a_{11}-a_{31} a_{22} a_{44} a_{13}+a_{24} a_{31} a_{42} a_{13}-a_{32} a_{41} a_{24} a_{13} \\
& -a_{22} a_{33} a_{41} a_{14}-a_{14} a_{23} a_{31} a_{42}+a_{32} a_{41} a_{23} a_{14}
\end{aligned}
$$

On remarque que (10) est une équation algébrique du quatrième degré. Pour connaître si l'état est stable ou instable, il faut déterminer ses racines.

En effet si les parties réelles des quatre racines de I 'équation caractéristique (10) sont positives, on dit que l'état $n_{0 s}, n_{t \mid s}, N_{a s}, N_{b s}$ est stable, au contraire si l'une au moins de ces racines possède un partie réelle négative, cet état sera instable.

La question de la stabilité ou d'instabilité de l'état stationnaire initial revêt une importance fondamentale pour établir si l'auto-amorçage d'oscillation peut se réaliser dans un laser à filtre saturable. Si l'état initial est stable dans ce cas, les fluctuations de la densité du champ et de la densité d'inversion de populations ne peuvent pas faire osciller le laser. Autrement dit, la stabilité de l'état stationnaire signifie que le pompage de l'élément actif est incapable à lui seul de faire passer le laser à filtre saturable au régime d'oscillation sans l'action d'une source secondaire.

\section{Conclusion}

L'analyse de la stabilité linéaire des solutions stationnaires nous a montré l'existence de deux solutions stables et par conséquent le phénomène de la bistabilité optique dans le cas où l'émission spontanée est négligée dans le système d'équation du bilan (R.A.E).

D'après les résultats obtenus dans l'analyse de la bistabilité optique, nous constatons que la branche représantant $Q_{j 1}$ est instable car elle a deux racines positives et une racine négative des valeurs propres $\lambda_{i}$ quelques soient les paramètres du L.S.A pris dans I' intervalle de la bistabilité optique. Par contre, les deux autres branches $Q_{j 0}$ et $Q_{j 2}$ sont stables. L'existence de ces deux solutions stables est à l'origine de la dénomination "bistabilité optique" donnée à ce phénomène.

\section{Références:}

[1] DINH VAN HOANG, TRAN THI THU HA, Inf.Phys., Volume 32, (1991) pp75-79

[2] DINH-VAN-HOANG; PHUNG-QUOC-BAO, Proceedings-of-the-SPIE-The-InternationalSociety-for-Optical-Engineering. 1999; 3862: pp 51-6

[3] PHUNG-QUOC-BAO; DINH-VAN-HOANG; VU-VAN-LUC, Journal-of-Russian-LaserResearch. July-Aug. 1999; 20(4): 307-16 
[4] PHYNG-QUOC-BAO and Al, Journal of Russian laser research. July-Aug 1999 20(4), pp297-306.

[5] S.DJABI, Thèse de magister IOMP, Université de Sétif (1993) [6] A.FRANCON, Thèmes actuels en optique, Edition Masson (1986)

[7] A.TARASSOV, Physique des processus dans les générateurs de rayonnement cohérent, Edition Mir (1985)

[8] M.TACHIKAWA, JOSA.B, Vol.4, N³, (1987) pp.387-394

[9] D.DANGOISSE et AL, Les Lasers - Cours et Exercices, Edition Dunod, Paris (1998) 